\title{
Quality standards in Biobanking: authentication by genetic profiling of blood spots from donor's original sample
}

\begin{abstract}
Sergio Cardoso ${ }^{1}$, Laura Valverde ${ }^{1}$, Adrian Odriozola ${ }^{1}$, Xabier Elcoroaristizabal $^{1}$ and Marian M de Pancorbo ${ }^{\star, 1}$
The field of Biobanking requires extensive work to maintain traceability of samples. However, sometimes the necessity to authenticate a sample may arise. To address these circumstances, we herein present a method for authenticating derivatives by using a blood spot from each donor, attached to a sample authentication form, by means of genetic profiling. Blood spots are collected at the time a blood sample is donated at a health centre and before processing the blood sample at the biobank. To test the validity of our approach over time, we analyzed 26 blood spots stored at room temperature in our facilities for more than 15 years. DNA was successfully extracted from the three storage materials tested in this study and 15 STR markers plus amelogenin were subsequently analyzed. The storage of a small blood spot attached to a sample authentication form proved to be efficient for genetic profiling and, therefore, may constitute a long-lasting (at least 15 years), cost-effective and effortless approach for genetic authentication of samples in biobanks.
\end{abstract}

European Journal of Human Genetics (2010) 18, 848-851; doi:10.1038/ejhg.2010.16; published online 17 March 2010

Keywords: biobanking; authentication; traceability; STR; microsatellite; genetic profile

\section{INTRODUCTION}

Traceability of samples constitutes one of the cornerstones in the emerging field of Biobanking, but there are divergent viewpoints within the scientific community regarding the way to implement it efficiently. The development of automatic and robotic procedures has partially helped to overcome this difficulty. Yet, even with the assistance of new high-tech procedures, a biobank may on occasion need to authenticate the origin of a particular sample. Whenever a doubt related to traceability arises in a biobank it is usually very difficult to check whether a sample does belong to a specific donor. In such a case, the biobank will not be able to certify that samples are correctly supplied to other biobanks or research groups. It is therefore crucial for biobanking activities to implement a consistent system to guarantee authentication of biological samples.

Genetic identification techniques used in the field of Forensic Genetics might be successfully applied in sample authentication because they provide very high power of discrimination (in the order of one in several thousand million). Currently, the most common method used for human genetic identification is based on microsatellite or short tandem repeat (STR) loci. ${ }^{1-3}$ By using less than $5 \mathrm{ng}$ of DNA, up to 15 autosomal STR loci, plus the amelogenin gender-determining marker can be simultaneously amplified by means of some commercial multiplex PCR kits with fluorescently labeled PCR primers, ${ }^{4}$ including the 13 STR loci of the CODIS system, ${ }^{5}$ which is used worldwide. The main drawback to this genetic profiling system arises when it is necessary to process a large amount of samples as this is an expensive, labor-intensive technique.
With the aim of avoiding a genetic profile analysis of every donor when the samples enter a biobank, the DNA Bank of the University of the Basque Country (UPV/EHU) has devised a cost-effective and reliable method for genetic authentication of biological samples through the collection of blood spots from the original donor. These blood spots are available for further analyses if authentication is required. This procedure is applicable to any derivative such as cell collections, biological fluids or DNA obtained from blood samples in biobanks.

\section{MATERIALS AND METHODS}

The procedure presented herein is based on the preservation of small blood spots in $10 \mathrm{~mm}$ discs of storage material. A first blood spot collected for authentication purposes is attached to a sample authentication form by health staff at the time the blood sample is donated and the informed consent is signed (Supplementary Figure S1). Once the blood sample and all associated information have been received at the biobank, an aliquot is collected from the blood sample to create a second blood spot, which is also attached to the sample authentication form to be used by the biobank as an internal control. To ensure the privacy of donors, each sample authentication form is linked to information associated with the corresponding donor and every derivative of the blood sample by means of barcode labels generated by a Laboratory Information Management System. Blood spots, which are suitably protected from any manipulation and/or contamination (Figure 1), can be stored at room temperatures for long periods of time.

The validation of our approach was performed by analyzing 26 blood spots of more than 15 years old stored at room temperature, all of them belonging to 12 people (named S01 to S12; see Table 1) who worked in our laboratory and who voluntarily agreed to participate in the study. Genetic profiles of each 


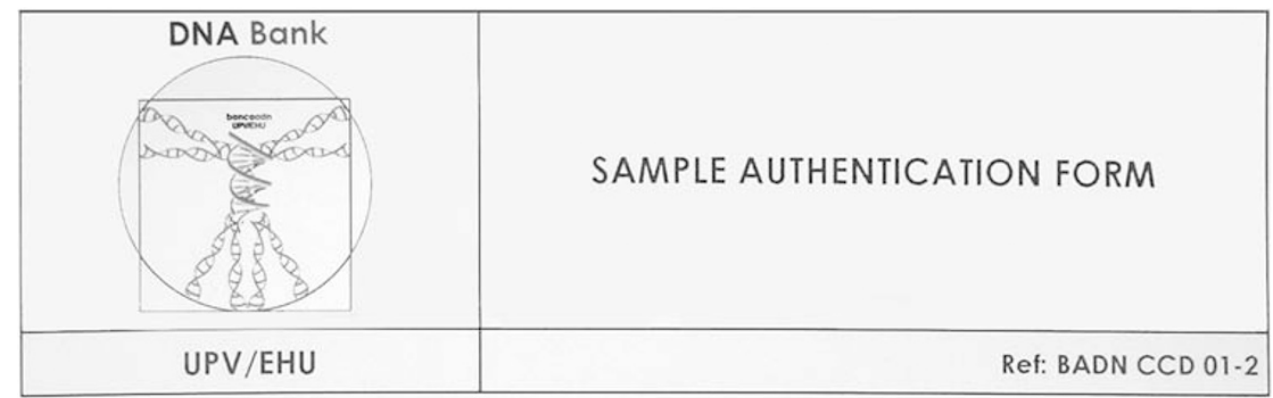

BARCODE AND BLOOD SPOTS

BARCODE / SAMPLE CODE

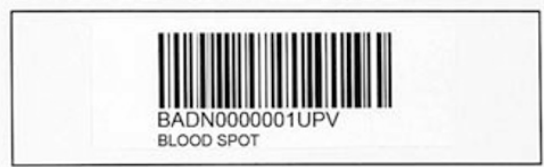

BLOOD SPOT (HEALTH CENTER)
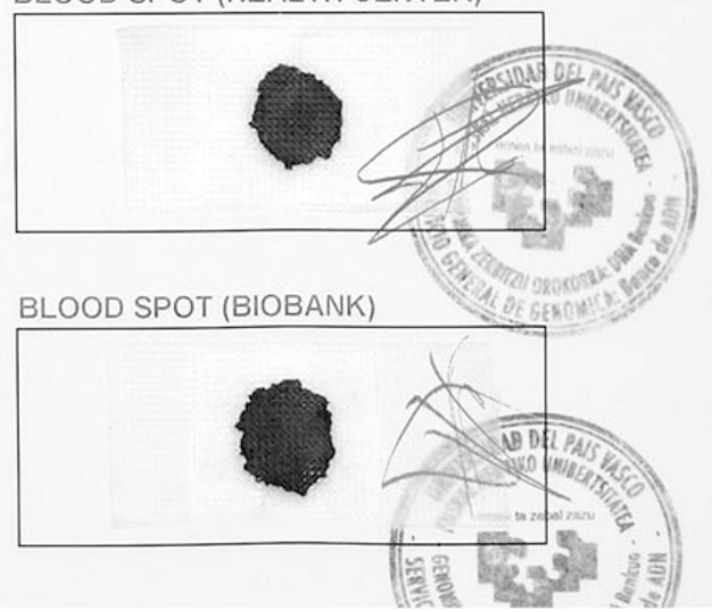

Figure 1 Blood spots attached to a sample authentication form covered with surgical tape. Blood spot collection at the time the sample is donated allows for genetic authentication of samples from the original donor. This collection is certified by the signatures of health staff. The signature of a member of the biobank staff validates the collection of the second blood spot when the sample enters the biobank; this blood spot would be used to trace sample processing in the laboratory. A rubber stamp allows further detection of any uncontrolled manipulation of blood spots.

Table 1 Genetic profiles obtained for all the samples analyzed in this study by means of the AmpFISTR Identifiler and SGM Plus kits ${ }^{a}$

Alleles observed in each locus

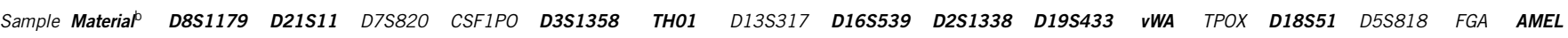

$\begin{array}{lllllllllllllllllllllllllllllllllllllllll}\text { S01 A, B, C, D } & 11 & 13 & 30 & 33.2 & 11 & 12 & 10 & 11 & 16 & 17 & 7 & 7 & 11 & 12 & 11 & 11 & 17 & 20 & 13 & 13 & 15 & 16 & 8 & 11 & 14 & 19 & 12 & 13 & 21 & 24 & \mathrm{X} & \mathrm{X}\end{array}$

$\begin{array}{llllllllllllllllllllllllllllllllllllll}\text { S02 } & \text { A, B, C, D } & 13 & 13 & 30 & 30 & 8 & 8 & 12 & 13 & 14 & 18 & 7 & 9.3 & 8 & 13 & 12 & 13 & 19 & 23 & 13 & 15.2 & 14 & 16 & 8 & 8 & 12 & 14 & 11 & 12 & 21 & 25 & \mathrm{X} & \mathrm{X}\end{array}$

$\begin{array}{lllllllllllllllllllllllllllllllllllllllll}\mathrm{S} 03 & \mathrm{~A}, \mathrm{~B}, \mathrm{C}, \mathrm{D} & 11 & 13 & 31 & 32.2 & 11 & 13 & 10 & 11 & 16 & 17 & 6 & 9.3 & 8 & 11 & 9 & 9 & 17 & 19 & 16 & 16.2 & 15 & 18 & 9 & 11 & 14 & 16 & 11 & 12 & 20 & 23 & \mathrm{X} & \mathrm{X}\end{array}$

$\begin{array}{llllllllllllllllllllllllllllllllllllllll}\mathrm{S} 04 & \mathrm{~A}, \mathrm{~B}, \mathrm{C}, \mathrm{D} & 13 & 13 & 30 & 30 & 8 & 11 & 11 & 12 & 14 & 18 & 9.3 & 9.3 & 9 & 13 & 11 & 12 & 19 & 23 & 13 & 13 & 14 & 16 & 8 & 8 & 11 & 13 & 11 & 12 & 23 & 25 & \mathrm{X} & \mathrm{X}\end{array}$

$\begin{array}{lllllllllllllllllllllllllllllllllllllllllll}\text { S05 } & \text { A, B, C, D } & 12 & 14 & 30 & 33.2 & 11 & 12 & 10 & 11 & 17 & 18 & 6 & 9 & 13 & 14 & 10 & 12 & 17 & 24 & 14 & 15 & 15 & 19 & 8 & 11 & 10 & 19 & 12 & 13 & 22 & 23 & \times & X\end{array}$

$\begin{array}{llllllllllllllllllllllllllllllllllllllllllll}\text { S06 } & \text { A, B, C, D } & 12 & 16 & 29 & 32.2 & 9 & 11 & 11 & 11 & 15 & 16 & 8 & 9 & 10 & 12 & 11 & 12 & 16 & 20 & 13 & 13 & 17 & 19 & 8 & 12 & 13 & 14 & 11 & 11 & 24 & 25 & \mathrm{X} & \mathrm{X}\end{array}$

$\begin{array}{lllllllllllllllllllllllllllllllllllllllllll}\text { S07 } & A, B, C & 12 & 15 & 28 & 30 & 10 & 11 & 10 & 12 & 15 & 17 & 6 & 9.3 & 11 & 11 & 11 & 11 & 17 & 19 & 15.2 & 16 & 15 & 18 & 8 & 8 & 14 & 16 & 11 & 11 & 19 & 26 & X & X\end{array}$

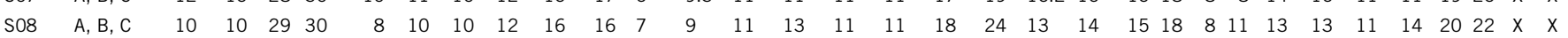

$\begin{array}{llllllllllllllllllllllllllllllllllllllll}\text { S09 } & \text { A, B } & 14 & 15 & 27 & 31 & 9 & 12 & 12 & 14 & 15 & 18 & 9.3 & 10 & 12 & 13 & 11 & 13 & 17 & 25 & 14 & 14 & 15 & 17 & 8 & 11 & 12 & 15 & 10 & 12 & 23 & 24 & \mathrm{X} & \mathrm{X}\end{array}$

$\begin{array}{llllllllllllllllllllllllllllllllllllllllll}\mathrm{S} 10 & \mathrm{~A}, \mathrm{~B} & 12 & 15 & 30 & 32.2 & 11 & 12 & 12 & 12 & 16 & 18 & 8 & 9.3 & 10 & 11 & 13 & 13 & 17 & 19 & 14 & 15 & 17 & 18 & 11 & 11 & 12 & 15 & 11 & 13 & 22 & 26 & \mathrm{X} & \mathrm{Y}\end{array}$

$\begin{array}{llllllllllllllllllllllllllllllllllllllllllllll}\mathrm{S} 11 & \mathrm{~A}, \mathrm{~B} & 12 & 14 & 30 & 31.2 & 8 & 11 & 10 & 11 & 15 & 15 & 7 & 9.3 & 8 & 11 & 11 & 13 & 20 & 24 & 13 & 15 & 16 & 17 & 11 & 12 & 15 & 15 & 9 & 12 & 19 & 24 & \mathrm{X} & \mathrm{X}\end{array}$

$\begin{array}{llllllllllllllllllllllllllllllllllllllll}\mathrm{S} 12 & \mathrm{~A}, \mathrm{~B} & 13 & 14 & 30 & 30 & 9 & 10 & 11 & 11 & 16 & 18 & 6 & 8 & 8 & 11 & 11 & 12 & 17 & 23 & 13 & 15 & 16 & 17 & 8 & 8 & 13 & 15 & 11 & 13 & 18 & 23 & \mathrm{X} & \mathrm{Y}\end{array}$

a Microsatellite loci highlighted in bold are those amplified within the AmpFISTR SGM Plus kit and also analyzed by means of the AmpFISTR Identifiler kit.

${ }^{b}$ Material used for preservation of each sample, A: DNA stored at $-20^{\circ} \mathrm{C}$; B: non-colored cotton fabric; C: lint; D: absorbent paper. 
participant used as reference in this study were obtained from DNA stored at $-20^{\circ} \mathrm{C}$. Six out of the 26 blood samples were preserved on absorbent paper, eight in a piece of lint and 12 stored on non-colored cotton fabric. The blood spots collected at the beginning of 1994 were at that time attached to the informed consent of each donor using surgical tape, as described by RodríguezAlarcón et al. ${ }^{6}$ Informed consents were stored at room temperature in a security cabinet, protected from sunlight and at variable moisture levels, according to the season.

Blood spot samples were retrieved for DNA extraction in Autumn 2008. We used a $3.0 \mathrm{~mm}$ Whatman Harris punch to obtain a portion of the storage material. This portion was subsequently cut into small pieces using sterile scissors. Samples were incubated overnight at $56^{\circ} \mathrm{C}$ in $500 \mu \mathrm{l}$ of lysis solution $(\mathrm{NaCl} 0.2 \mathrm{M}$, EDTA $10 \mathrm{~mm}$, Tris $10 \mathrm{~mm}$, SDS $2 \%$, DTT $40 \mathrm{~mm}$ and $0.8 \mathrm{mg} / \mathrm{ml}$ proteinase $\mathrm{K}$ as final concentration). DNA extraction was performed using the standard phenol-chloroform procedure. ${ }^{7}$ First, $200 \mu \mathrm{l}$ of phenol/chloroformisoamyl alcohol in a proportion of 25:24:1 were added to each tube. After centrifugation and recovery of the aqueous phase, two additional washes with chloroform-isoamyl alcohol were carried out to further purify the samples. Once the aqueous phase was recovered, we added $\mathrm{NaAc} 2 \mathrm{M}$ in a proportion of $1 / 10$ to the volume of aqueous phase, $1 \mu \mathrm{l}$ of glycogen $(10 \mathrm{mg} / \mathrm{ml})$ and two volumes of cold absolute ethanol $\left(-20^{\circ} \mathrm{C}\right)$. To facilitate DNA precipitation, sample tubes containing the precipitation mix were stored at $-20^{\circ} \mathrm{C}$ for $2 \mathrm{~h}$. Subsequent centrifugation at $14000 \mathrm{~g}$ was carried out to obtain the DNA pellet; this pellet is frequently not visible when extracting DNA from old blood spots. After a final wash with EtOH (70\%), samples were introduced into a centrifugal evaporator (DNA mini; Heto Holten, Allerød, Denmark) to get the pellet dried. Finally, samples were resuspended in $20 \mu \mathrm{l}$ of sterile water.

The Quant-iT PicoGreen dsDNA Assay Kit (Invitrogen, Carlsbad, CA, USA) was used to quantify the extracted DNA. We added $1 \mu \mathrm{l}$ of each sample to $199 \mu \mathrm{l}$ of the quantification mix (1/400 PicoGreen and 1/20 Buffer TE $20 \times$ ) and DNA concentration was obtained in a VersaFluor fluorometer (BioRad, Richmond, CA, USA). Genetic profiles of microsatellite loci for each sample (including reference samples) were obtained by means of PCR amplification in a GeneAmp 9700 Gold (Applied Biosystems, Foster City, CA, USA) using two commercial kits: AmpFISTR Identifiler PCR Amplification Kit and AmpFISTR SGM Plus PCR Amplification Kit (Applied Biosystems). Only 2 ng of DNA were used for each amplification reaction. Fragment analysis of PCR products was carried out by capillary electrophoresis using an ABI3130 Genetic Analyzer (Applied Biosystems). Finally, GeneMapper Software v4.0 (Applied Biosystems) was used for allele designation.

We conducted a one-way analysis of variance (ANOVA) followed by a Tukey-Kramer multiple-comparison post hoc test to determine whether mean DNA concentrations obtained for the three different storage materials showed significant differences.

\section{RESULTS}

The blood spots stored in the three different support media (absorbent paper, lint or non-colored cotton fabric) did not show notable degradation signs, irrespective of the material used. Similarly, oscillations of moisture levels over years did not seem to affect blood spot conservation, because no fungal growth was observed in any of the samples.

DNA extraction from blood spots was successful in the three types of storage material tested in our study. Nevertheless, DNA extraction from lint proved to be somewhat complicated because it was rather difficult to manipulate this kind of material, particularly when we attempted to cut it into smaller pieces to facilitate the extraction procedure. Subsequent DNA quantification with PicoGreen confirmed DNA isolation: all the samples rendered concentrations that varied between 1.5 and $28 \mathrm{ng} / \mu \mathrm{l}$ of double-strand DNA. Mean DNA concentrations $( \pm \mathrm{SE}$ ) obtained for lint, absorbent paper and cotton fabric were $7.3 \pm 1.6,14.8 \pm 3.1$ and $13.2 \pm 1.8 \mathrm{ng} / \mu \mathrm{l}$, respectively. Significant differences between the DNA concentrations obtained in each material were revealed by the ANOVA results $(\mathrm{F}=4.36$; d.f. $=2,25$; $P<0.05)$. The Tukey test showed no differences between the DNA concentrations of cotton fabric and absorbent paper, which in turn differed from the concentration obtained in lint $(P<0.05)$.

In obtaining the STR profiles, 2 ng of DNA per sample and commercial kit were employed. Table 1 shows the genetic profile obtained for each sample with both commercial kits and the comparison with the corresponding reference profile. Complete and reliable genetic profiles were obtained for each one of the 26 blood spots included in the analysis. Allele genotyping profiles obtained for each sample with the two different commercial kits used were coincident for all common STR markers, as shown in Supplementary Figure S2 for sample S01. This double-checking system constitutes a noteworthy validation step of the method proposed herein. In this sense, no allelic drop-out due to DNA degradation was detected in the 15-year-old samples examined, and even fragments of the STR markers with the largest sizes were correctly amplified. Further evidence of the reliability and validity of our method was the fact that the genetic profiles obtained for each sample exactly matched with the genetic profile of the corresponding individual participating in this study.

\section{DISCUSSION}

Authentication procedures are fundamental to biobanking services, because they must ensure that a specific sample or set of samples come from a specific donor before supplying them to the scientific community. Therefore, biobanks are required for preventing and identifying errors, such as sample misplacement. In fact, an emphasis on high standards in traceability is recommended by many European initiatives for the international networking of Biobanking. ${ }^{8-10}$ Genetic profiling seems to be an efficient approach for sample authentication. Interestingly, there are very few studies focusing on authentication procedures in biobanks by means of genetic profiling, most of them centered on cell line authentication. ${ }^{1-14}$ Indeed, obtaining the genetic profile for the whole collection of a biobank might be extremely expensive and laborious. To overcome this limitation, we have devised a system based on the conservation of blood spots that allows a biobank to resort to using the genetic profile of the donor only when strictly necessary. The collection of the first blood spot at the time a blood sample is donated at a health centre is crucial to carry out our method of authentication. Thus, this blood spot would allow authentication of any derivative to the original donor, whereas the second blood spot would be used to trace sample processing at the biobank as a kind of internal control.

There are various commercial systems for fluid storage at room temperature, such as FTA cards (Whatman) or IsoCode DNA matrix (Schleicher \& Schuell BioScience Inc.). Yet, biobanks often have to operate within a limited budget in a relatively expensive field and, therefore, implementing low-cost systems may be a valid alternative. In this sense, all the storage materials tested in this study proved to be effective for the conservation of blood spots at room temperature for long periods of time. However, taking into account the DNA concentration obtained for each substrate, non-colored cotton fabric and absorbent paper seem to be most effective for long-term conservation. It is likely that cotton strands in lint form an excessively open net, in which the DNA is more accessible by any degrading agent. It might be advisable to analyze a larger sample set to replicate these results. However, even though the DNA Bank of the UPV/EHU accumulates a very large number of samples, only the blood spots from the 12 individuals included in this study were stored exactly in the conditions presented herein and for a period of more than 15 years.

The method of genetic profiling presented herein involves the use of two commercial kits. Two main reasons led us to select these kits: (1) the SGM Plus Kit shares its 10 STR markers with 10 out of 15 
microsatellite markers of the Identifiler PCR Amplification Kit. Therefore, the genetic profile of each sample is partially double checked, thereby avoiding a potential error in the designation of alleles caused by allelic drop-out due to DNA degradation and, at the same time, improving the quality and reliability of the results; and (2) the use of standardized and validated commercial kits for STR genotyping provides the possibility of managing comparable data ${ }^{15}$ between biobanks and conducting inter-laboratory quality controls. In addition, the genetic profiling step would not routinely be carried out, so that the cost increase is not expected to be high.

In cases where misplacement is detected, our approach would allow the biobank to confirm a putative traceability gap and, once confirmed, to undertake appropriate corrective actions. It would be advisable to analyze all the derivatives related to the affected sample (plasma, DNA, cell lines, etc.). Thus, the confirmation of a mismatch would involve, at the very least, discarding the 'orphan' derivatives from the biobank collection and, therefore, stopping the supply of incorrectly stored derivatives.

Concerning the legal and ethical aspects involving sample genotyping for authentication purposes, each biobank must follow the governing laws in the corresponding country. ${ }^{16}$ The blood preservation method presented herein complies both with Spanish biomedical research law $14 / 2007^{17}$ on the traceability of data and biological samples and Organic Law $15 / 1999^{18}$ on personal data protection. According to these laws, all the information related to each sample should be kept at the biobank as classified information. In compliance with these laws, we have developed a sample authentication form specifically designed to store blood spots for long periods of time. This method makes it possible to maintain the anonymity of the donor and, at the same time, make use of a blood spot from the original sample for authentication purposes. To further comply with ethical recommendations about information to donors, notification about the collection and storage of blood spots for sample authentication should be included as part of the informed consent.

Furthermore, genetic data generated during the authentication process should be stored at biobanks, bearing in mind that they take responsibility for all data resulting from the collection, storage, processing and shipment of each sample. However, in compliance with the governing laws in many countries such as Spain, upon a donor's request, a biobank is obliged to destroy the sample and all its derivatives. Our approach conforms to legal requirements as barcode labels generated during sample processing allow us to identify and discard all components of any sample, including any authentication forms.

To summarize, the results of this study obtained from samples which are years old indicate that the storage of small spots of dried blood preserved at room temperature in $10 \mathrm{~mm}$ discs of non-colored cotton fabric or absorbent paper, attached to a sample authentication form and conveniently protected from mishandling and/or contamination, could be a long-lasting (at least 15 years), cost-effective and effortless approach for subsequent genetic authentication of biobank samples. In addition, any analysis of genetic profiles through use of commercial, standardized kits for STR typing provides for the quality requirements of a biobank, which is an issue of vital importance in the up-and-coming field of biobank networking.

\section{CONFLICT OF INTEREST}

The authors declare no conflict of interest.

\section{ACKNOWLEDGEMENTS}

This work was partially funded by the Basque Government (IT-424/07). We thank the Servicios Generales de Investigación (SGIker) of the University of the Basque Country and Fundación Genoma España for their support.

1 Gómez J, Carracedo A: The 1998-1999 collaborative exercises and proficiency testing program on DNA typing of the Spanish and Portuguese Working Group of the International Society for Forensic Genetics (GEP-ISFG). Forensic Sci Int 2000; 114: 21-30.

2 Butler JM (eds): Forensic DNA Typing: Biology, Technology, and Genetics of STR Markers. Academic Press: New York, 2005, 2nd edn.

3 Butler JM: Genetics and genomics of core short tandem repeat loci used in human identity testing. J Forensic Sci 2006; 51: pp 253-265.

4 Wallin JM, Holt CL, Lazaruk KD, Nguyen TH, Walsh PS: Constructing universal multiplex PCR systems for comparative genotyping. J Forensic Sci 2002; 47: 52-65.

5 Miller KW, Brown BL, Budowle B (eds): The Combined DNA Index System; in International Congress Series 1239. Elsevier, Amsterdam, New York, 2003, pp 617-620.

6 Rodríguez-Alarcon J, de Pancorbo MM, Santillana L et al: La Huella De ADN En Lugar De La Huella Plantar En La Identificacion Neonatal. Medicina Clinica 1996; 107: 121-123.

7 Sambrook J, Fritsch EF, Maniatis T: Molecular cloning: A laboratory manual. Cold Spring Harbor Laboratory Press: Cold Spring Harbor, 1989, 2nd edn.

8 Asslaber M, Zatloukal K: Biobanks: transnational, European and global networks. Brief Funct Genomic Proteomic 2007; 6: 193-201.

9 Yuille M, van Ommen GJ, Bréchot $\mathrm{C}$ et al: Biobanking for Europe. Brief Bioinform 2008; 9: 14-24.

10 Public Population Project in Genomics (P3G).

11 Gilbert DA, Reid YA, Gail MH et al: Application of DNA fingerprints for cell-line individualization. Am J Hum Genet 1990; 47: 499-514.

12 Stacey GN, Bolton BJ, Doyle A: DNA fingerprinting transforms the art of cell authentication. Nature 1992; 357: 261-262.

13 Masters JR, Thomson JA, Daly-Burns B et al: Short tandem repeat profiling provides an international reference standard for human cell lines. Proc Natl Acad Sci 2001; 98: 7656-7658.

14 Azari S, Ahmadi N, Tehrani MJ, Shokri F: Profiling and authentication of human cell lines using short tandem repeat (STR) loci: Report from the National Cell Bank of Iran. Biologicals 2007; 35: 195-202.

15 Chakraborty R, Stivers DN, Su B, Zhong Y, Budowle B: The utility of short tandem repeat loci beyond human identification: Implications for development of new DNA typing systems. Electrophoresis 1999; 20: 1682-1696.

16 Wallace S, Bédard K, Kent A, Knoppers BM: Governance mechanisms and population biobanks: Building a framework for trust. GenEdit 2008; 6: 1-11.

17 Ley de Investigación Biomédica BOE no. 159; 4 July 2007, pp 28826-28848 [www.boe.es/diario boe/].

18 Ley Orgánica 15/1999 de Protección de Datos de Carácter Personal BOE no. 29814 December 1999, pp 43088-43099 [www.boe.es/diario_boe/].

Supplementary Information accompanies the paper on European Journal of Human Genetics website (http://www.nature.com/ejhg) 